\title{
Die varisierende distale Femurosteotomie mit dem TomoFix-Plattenfixateur zur Behandlung der Valgusgonarthrose
}

\author{
$\square$ Denise Freiling, Ronald van Heerwaarden, Philipp Lobenhoffer
}

\section{Zusammenfassung}

Kniegelenksnahe Osteotomien erleben in den letzten Jahren eine wahre Renaissance. Dies ist zum einen auf neue Implantate, welche eine weitgehend funktionelle Nachbehandlung zulassen, wie auch auf verbesserte schonende Operationstechniken zurückzuführen. Eingriffe an der proximalen Tibia zur Behandlung von Varusgonarthrosen gehören aufgrund dieser Neuerungen mittlerweile in vielen orthopädischen und traumatologischen Einrichtungen zu erfolgreichen Standardoperationen $[5,6]$. Obwohl Langzeitergebnisse zum heutigen frühen Zeitpunkt noch nicht erhältlich sind, motivieren zufriedene Patienten zu weiteren Innovationen. Während die operative Korrektur der O-Bein-Fehlstellung in vielen Publikationen sehr detailliert beschrieben ist [2], wird die Behandlung von X-Bein-Deformitäten auch heute noch oftmals kontrovers diskutiert. Da die Fehlstellung zumeist im Bereich des distalen Femurs zu finden ist, sollte die operative Behandlung auch in diesem Bereich durchgeführt werden. In der Literatur der letzten Jahre werden viele Verfahren beschrieben $[1,3,4,8,10]$. Dort ist u.a. von lateral-öffnenden Techniken oder von Domosteotomien zu lesen. Häufig wurden hierbei jedoch sowohl lokale Beschwerden durch die einzelnen Fixationsmethoden (Weichteilirritationen durch das Osteosynthesematerial) als auch Wundheilungsstörungen oder verzögerte Knochenheilungen beobachtet und somit der Verlauf deutlich verzögert.

\section{Distalmedial Closed Wedge Osteo- tomy of the supracondylar Femur with a Special Internal Plate Fixator (TomoFix) for Treatment of Valgus Deformity of the Lower Limb}

The goal of distal medial closed wedge femur osteotomy in patients with unicompartimental osteoarthritis of the lateral compartment of the knee is to shift the mechanical leg axis from the lateral to the medial compartment. There are many causes for lateral osteoarthritis. Patients with total or subtotal resections of the lateral meniscus often develop severe osteoarthritis of the lateral compartment in combination with a valgus deformity of the lower limb. In the past years, various possibilities for surgical corrections have been developed, e.g., domeshaped osteotomies or open wedge lateral osteotomies of the distal femur. Unfortunately, local complaints have occurred in many cases, mostly caused by the fixation methods (e.g., irritations of the tractus iliotibialis) or complications in wound healing or delayed bone healing. We now present a new improved surgical technique with a special internal plate fixator.
Vor diesem Hintergrund wird im Folgenden eine verbesserte Operationstechnik (inkomplette closed-wedge Osteotomie) mit einem speziell für diesen Eingriff konzipierten internen Plattenfixateur beschrieben [11].

\section{Ätiologie der Valgusgonarthrose}

Die monokompartimentale laterale $\mathrm{Ar}$ throse im Knie entsteht im Allgemeinen nach totalen oder partiellen Resektionen des Außenmeniskus. Der Meniskus dient u.a. als Puffer zwischen dem Femur und der Tibia. Wird dieser Puffer geschädigt kommt es häufig zu Knorpelschädigungen im äußeren Gelenkabschnitt, welche in fortgeschrittenen Stadien zu einer sekundären Valgusabweichung der unteren Extremität führen können.

Weitaus seltener ist die X-Bein Deformität bereits angeboren oder posttraumatisch nach einer Fraktur entstanden.

In den meisten Fällen ist die Fehlstellung im Bereich des distalen Femurs lokalisiert. In wenigen Fällen kann jedoch eine tibiale Deformität vorliegen. Im Allgemeinen sollte immer im Bereich der Fehlstellung korrigiert werden.

Liegt eine tibiale Achsabweichung vor, so sollte die operative Korrektur an der Tibia erfolgen, um iatrogene Fehlstellungen, welche mit einer schrägen Gelenkslinie einhergehen, zu vermeiden. Selten sind komplizierte Doppelosteotomien an Tibia und Femur notwendig. Der Korrekturoperation sollte in allen Fällen eine genaue Analyse der Fehlstellung vorausgehen. Hierzu müssen einige Winkel im Bereich der gesamten unteren Extremität bestimmt werden. Wir empfehlen die Prinzipien der Deformitätenchirurgie von D. Paley genauestens zu beachten [8].

Präoperativ ist eine exakte radiologische Planung des Korrekturausmaßes mittels einer Ganzbeinaufnahme unerlässlich (Abb. 1). 


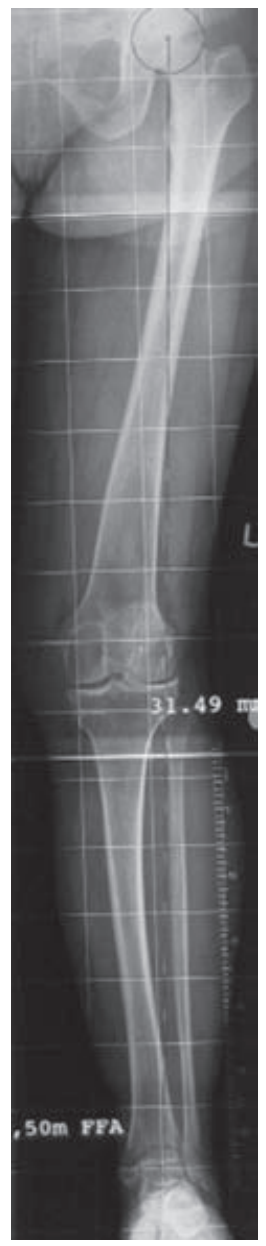

Abb. 1 Die Ganzbeinaufnahme (inkl. Hüftkopzentrum und Mitte des oberen Sprunggelenkes) ist essenziell für die präoerative Planung der Umstellungsosteotomie.

Die in vielen Abteilungen erstellten „langen Aufnahmen“ sind als alleinige Planungshilfe obsolet, da sowohl das Hüftkopfzentrum als auch die Mitte des oberen Sprunggelenkes nicht berücksichtigt werden können. Wir verwenden die zeichnerische Planungsmethode nach Miniaci [9], welche sowohl an der digitalen Röntgenkonsole (Abb.2a und $\mathbf{b}$ ) als auch manuell angefertigt werden kann. Dabei sollte die postoperative mechanische Tragachse bei Patienten mit einer lateralen Arthrose und Valgusmorphotyp in etwa durch den medialen Eminentiahöcker verlaufen. Patienten mit valgischer Beinachse ohne höhergradige Knorpelläsionen benötigen hingegen eine weniger ausgeprägte Korrektur. In unserer Abteilung wird in diesen Fällen eine femoro-tibiale Achse von $0^{\circ}$ (Mitte des Knies) angestrebt.

\section{Präoperative Vorbereitungen}

Neben der exakten körperlichen Untersuchung, welche neben der Weichteilsituation besonders die Bandspannung des Kniegelenkes berücksichtigt, sollte zusätzlich zu der Ganzbeinaufnahme eben-
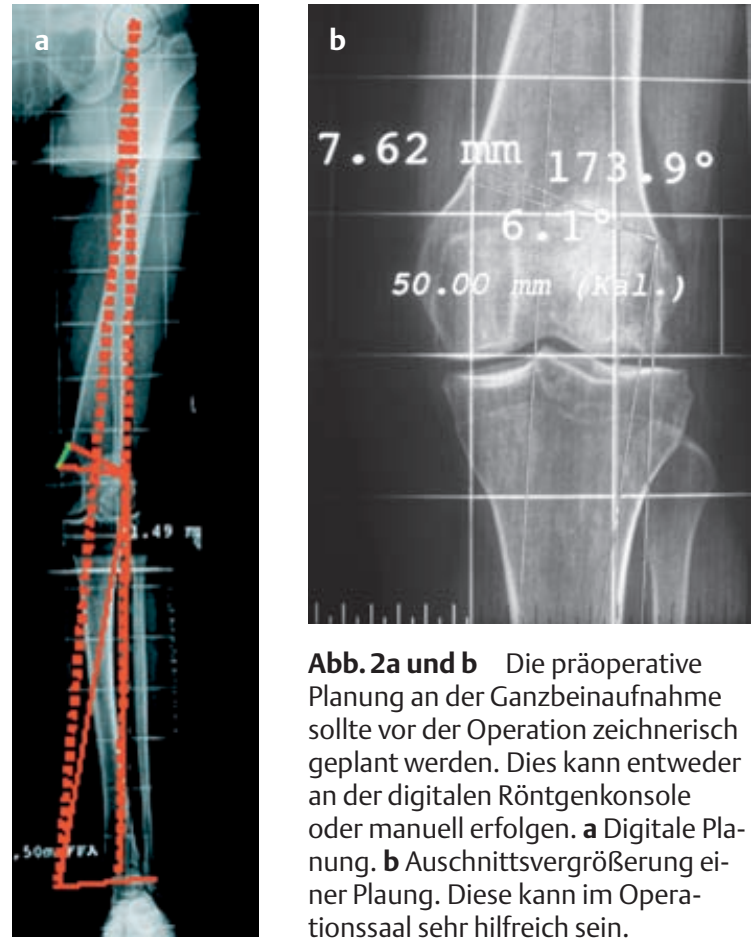

Abb. 2a und b Die präoperative Planung an der Ganzbeinaufnahme sollte vor der Operation zeichnerisch geplant werden. Dies kann entweder an der digitalen Röntgenkonsole oder manuell erfolgen. a Digitale Planung. b Auschnittsvergrößerung einer Plaung. Diese kann im Operationssaal sehr hilfreich sein.

falls Röntgenbilder des betroffenen Knies in 2 Ebenen durchgeführt werden. MRTBilder zur Beurteilung des Gelenkstatus (besonders im Bereich des medialen Kompartimentes) können hilfreich sein, sind aber in unserer Abteilung nicht zwingend notwendig. Wir führen unmittelbar vor jeder Umstellungsoperation eine Arthroskopie des Kniegelenkes durch. Dies hat den Vorteil, dass neben der Erhebung des Gelenkstatus, ebenfalls arthroskopische Operationen (Außenmeniskusteilresektionen, Mikrofrakturierung etc.) durchgeführt werden können. Von großer Bedeutung ist das ausführliche präoperative Gespräch mit dem Patienten, welches im Idealfall vom Operateur selbst durchgeführt werden sollte. Hierbei muss neben den allgemeinen operativen Risiken (Infektion, Gefäß-, Nervenschäden etc.) unbedingt besprochen werden, dass in manchen Fällen erst nach ca. 4-5 Monaten eine deutliche Beschwerdelinderung eintreten kann. Dieses ist auf die häufig zögerliche Anpassung des Körpers zurückzuführen.

\section{Indikationen und Kontraindikationen}

Der ideale Patient für die varisierende distale closed-wedge Osteotomie mit dem TomoFix ${ }^{\mathrm{TM}}$ distales mediales Femur besitzt einen Valgusmorphotyp in Kombination mit einer unikompartimentalen lateralen Gonarthrose. Der Patient sollte 60 Jahre und einen BMI von 30 nicht überschreiten. Anderenfalls wurde die
Zunahme von Pseudarthrosen und Wundheilungsstörungen beobachtet. Ein gewisser Aktivitätsgrad des Patienten wirkt positiv beeinflussend auf die Erfolgsrate der Operation. Studien haben gezeigt, dass der Nikotinabusus hingegen die Knochenheilung entscheidend kompromittiert und das Infektionsrisiko deutlich erhöht. Die präoperative Kniegelenksbeweglichkeit sollte für die Flexion mindestens 90 Grad betragen. Ein geringes Streckdefizit ist tolerabel und kann gegebenenfalls intraoperativ korrigiert werden. Als Kontraindikationen gelten höhergradige Knorpelschäden (< III. Grades nach Outerbridge [7]) im Bereich des medialen Gelenkkompartimentes, der Verlust des Innenmeniskus sowie Adipositas $(\mathrm{BMI}<30)$. Bei einer höhergradigen Einschränkung der Beweglichkeit (Flexion < 90 Grad bzw. Streckdefizit>20 Grad) sowie einer mangelnden Patientencompliance sollte der Eingriff ebenfalls nicht durchgeführt werden. Präoperativ sind intakte Weichteilverhältnisse im Operationsgebiet von entscheidender Bedeutung.

Indikationen und Vorraussetzungen

1. Valgusmorphotyp

2. mediales Kompartment intakt

3. aktiver Patient

4. hohe Compliance

5. Alter: $<60$ lahre

6. Gewicht: BMI < 30

7. gute Beweglichkeit 
Kontraindikationen

1. Verlust des Innenmeniskus

2. Knorpelläsion $>I I^{\circ}$ nach Outerbridge mediales Kompartment

3. Adipositas (BMI über 30)

4. Nikotinabusus

5. höhergradige Bewegungseinschränkungen

\section{Operationstechnik}

Die varisierende distale closed-wedge Osteotomie des distalen Femurs kann in Allgemein- oder in rückenmarksnaher Regionalanästhesie durchgeführt werden. Die Operation wird in Rückenlage des Patienten durchgeführt. Das betroffene Bein sollte frei gelagert werden, sodass die Durchleuchtung von Hüfte, Knie und Sprunggelenk intraoperativ möglich ist (Abb.3). Eine sterile Blutsperre kann bedarfsweise angebracht werden. Dabei sollte das Operationsgebiet nicht eingeschränkt werden. Präoperativ wird eine Single-Shot-Antibiose verabreicht. Der Bildverstärker der intraoperativen Durchleuchtungskontrolle wird auf der Seite des zu operierenden Beines platziert. Der Operateur steht auf der Innenseite der Extremität.

\section{Instrumentarium}

Neben dem gängigen Instrumentarium der Knochenchirurgie wird der TomoFixMDF-Fixateur mit bi- und monokortikalen Kopfverriegelungsschrauben (Abb.4a und b), eine oszillierende Säge mit breitem Sägeblatt und $90 \mathrm{~mm}$ Länge, eine Sägelehre bzw. Kischner-Drähte zur Markierung der Osteotomie, ein chirurgischer Bildverstärker, eine sterile Metallstange zur Prüfung der Beinachse und eine sterile Messlehre zur Beurteilung der Höhe des Osteotomiekeils benötigt.

\section{Operationstechnik}

Die Operation wird zunächst in Extensionsstellung des Kniegelenks durchgeführt. Die anatomischen Landmarken werden auf der Haut markiert (Abb.5). Der Hautschnitt beginnt antero-medial, ca. eine Handbreit über dem oberen Patellapol und reicht distal bis zum oberen Drittel der Patella.

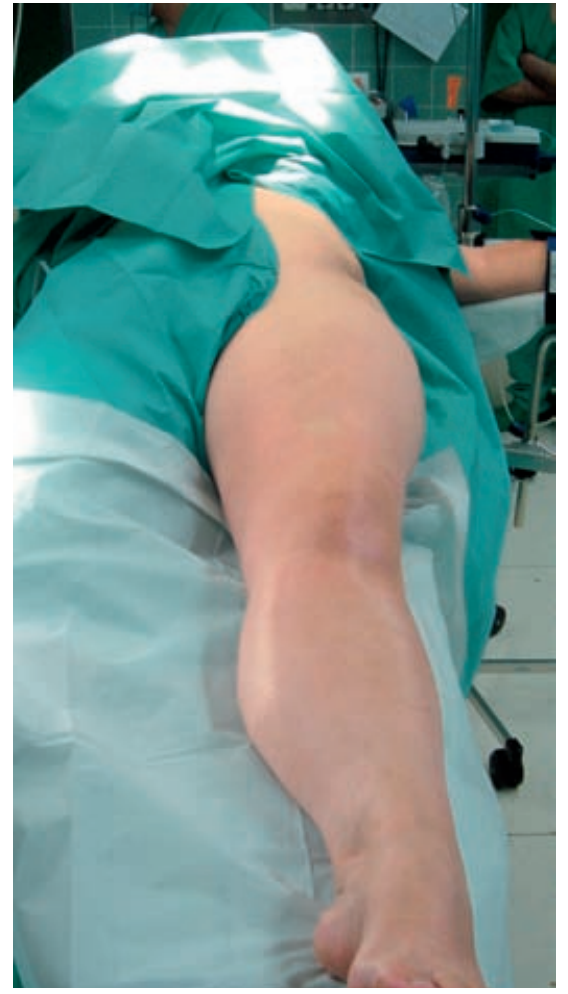

Abb. 3 Bei der Lagerung des Patienten sollte sowohl die Hüfte als auch das obere Sprunggelenk für den Operateur frei erreichbar sein.

Dieser Zugang kann bei nachfolgenden Operationen (z. B. Knie-TEP) erneut genutzt bzw. erweitert werden, wodurch Wundheilungsstörungen reduziert werden.

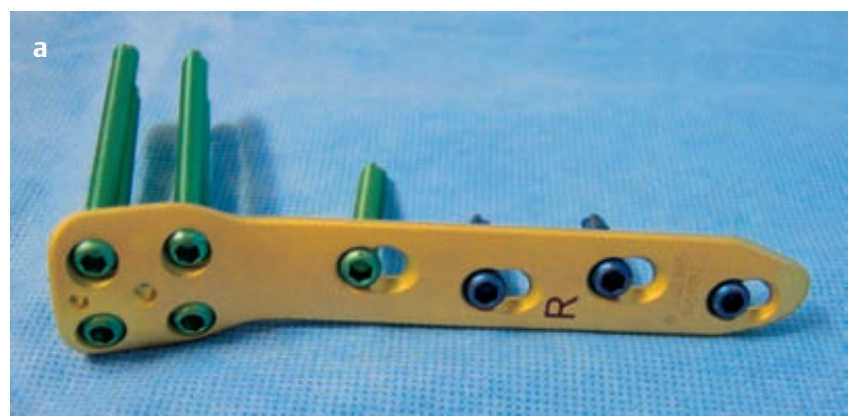

Abb. 4a und $b$ Der TomoFix MDF (MDF: mediales distales Femur) ist speziell für die schließende Osteotomie am Femur entwickelt worden.

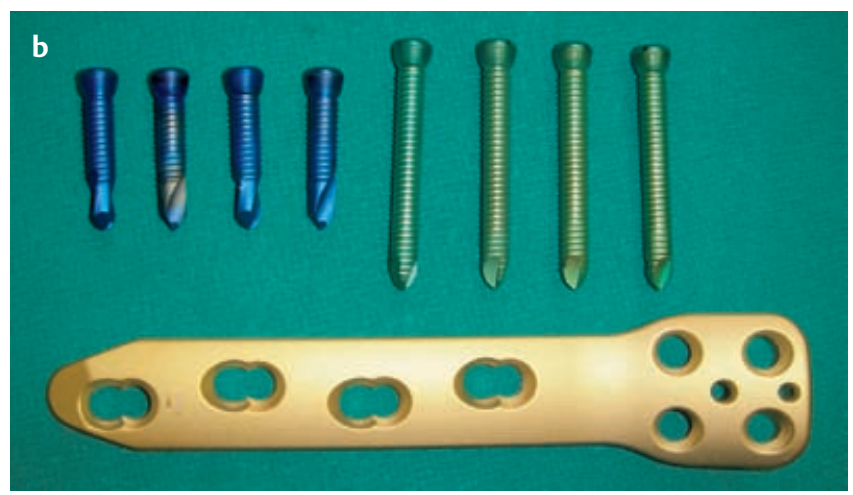




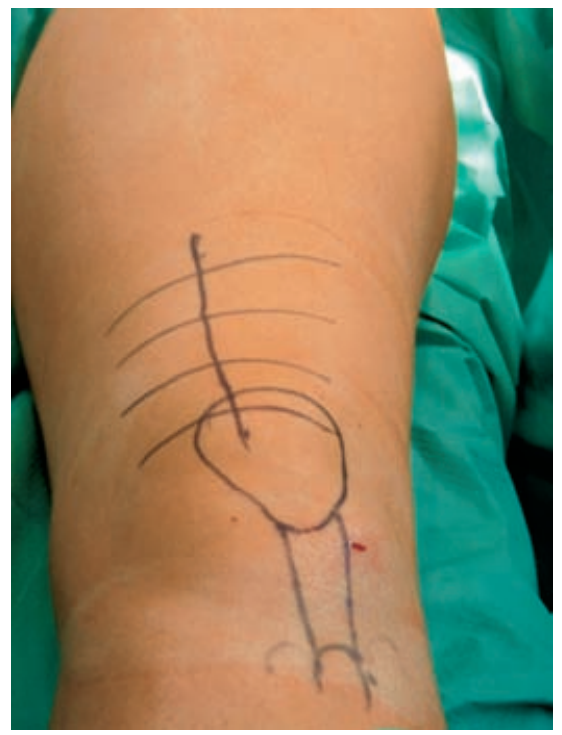

Abb.5 Vor dem Hautschnitt sollten die anatomischen Landmarken markiert werden.

Die K-Drähte dienen gleichzeitig als Führung der Säge. Die Osteotomien sollten unter ständiger Kühlung und Spülung mit einem neuen Sägeblatt durchgeführt werden. Im hinteren Anteil des Femurs muss auf einen sicheren Schutz der Weichteile und Gefäße geachtet werden. Im Anschluss wird der osteotomierte Keil entfernt (Abb.11). Der entnommene Keil wird mit der präoperativen Planung verglichen.

Zu diesem Zeitpunkt können noch Änderungen der Osteotomie vorgenommen werden, um eine Unterkorrektur zu vermeiden.

Die Osteotomie wird nun vorsichtig und kontinuierlich durch Druck auf den Fuß unter gleichzeitiger Stabilisierung der Kniegelenksregion geschlossen. Dies kann einige Minuten in Anspruch nehmen, bis durch plastische Verformung der lateralen Kortikalis der Schluss des Osteotomiespaltes erreicht ist. Die Osteotomie kann temporär durch zwei gekreuzte K-Drähte unter Berücksichtigung der künftigen Plattenlage fixiert werden. Nun wird die Ausrichtung der mechanischen Tragachse des Beines überprüft, indem eine lange Metallstange zwischen der radiologisch bestimmten Hüftkopfmitte und der Mitte des oberen Sprunggelenkes platziert wird (Abb.12a und b). Die Traglinie sollte entsprechend der präoperativen Planung verlaufen.

Nachdem der TomoFix MDF mithilfe der Positionierungshilfe mit den Bohrhülsen besetzt wurde (Abb.13), wird die Platte

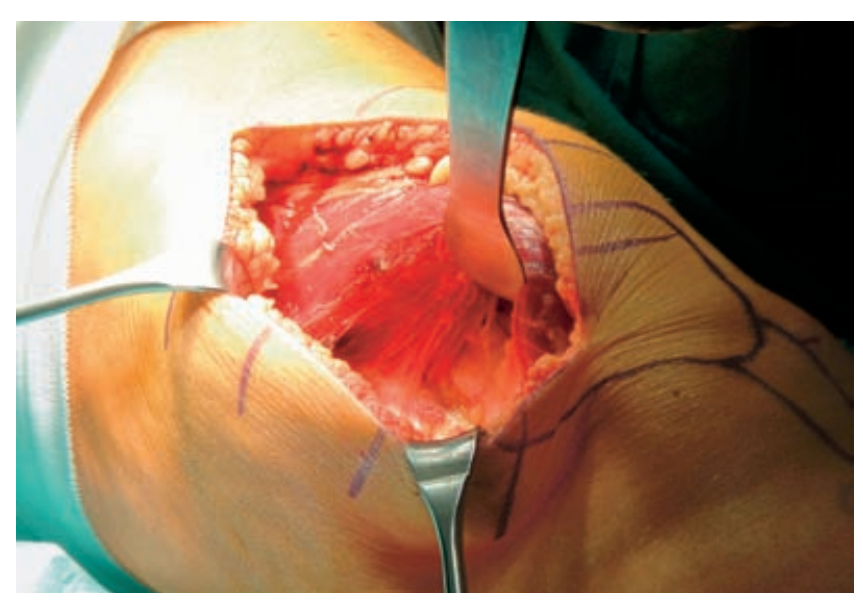

Abb. 6 Um den antero-medialen Aspekt des Oberschenkelknochens einzusehen, wird mit einem HohmannHaken die gesamte Muskulatur umfahren und nach streckseitig weggehalten.

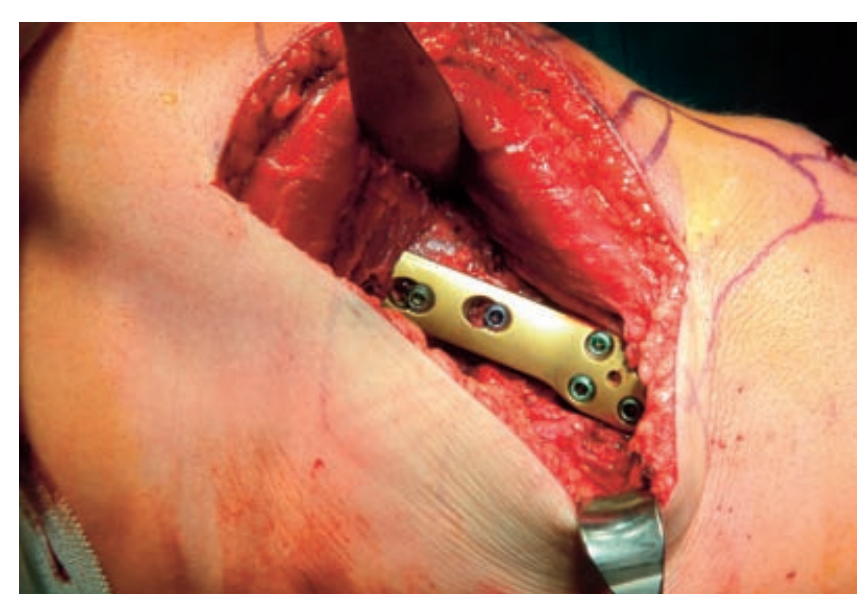

Abb.7 Der interne Plattenfixateur TomoFix MDF wird auf dem Knochen aufgelegt.

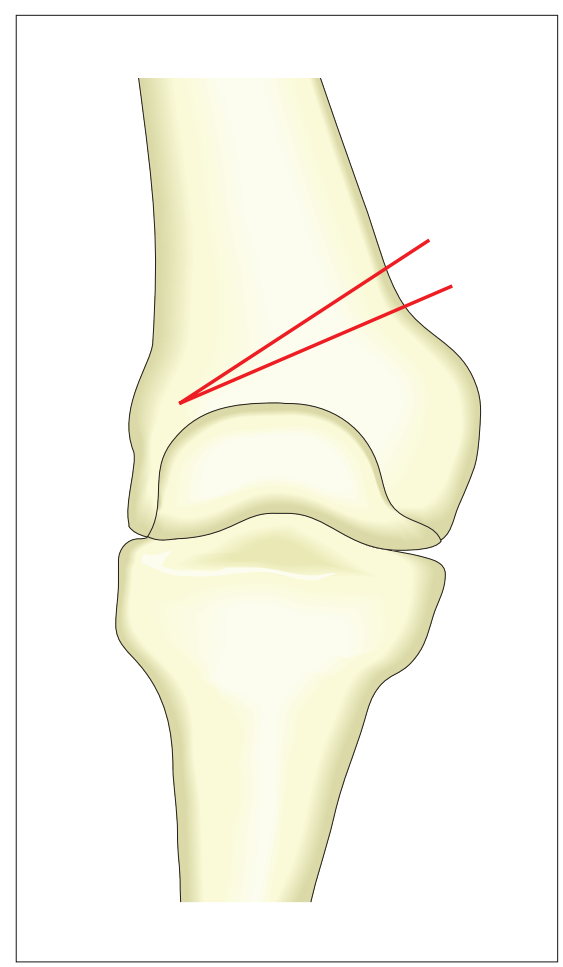

Abb. 8 Die schrägverlaufende Osteotomie beginnt medial in der suprakondylären Zone und endet innerhalb der lateralen Femurkondyle.

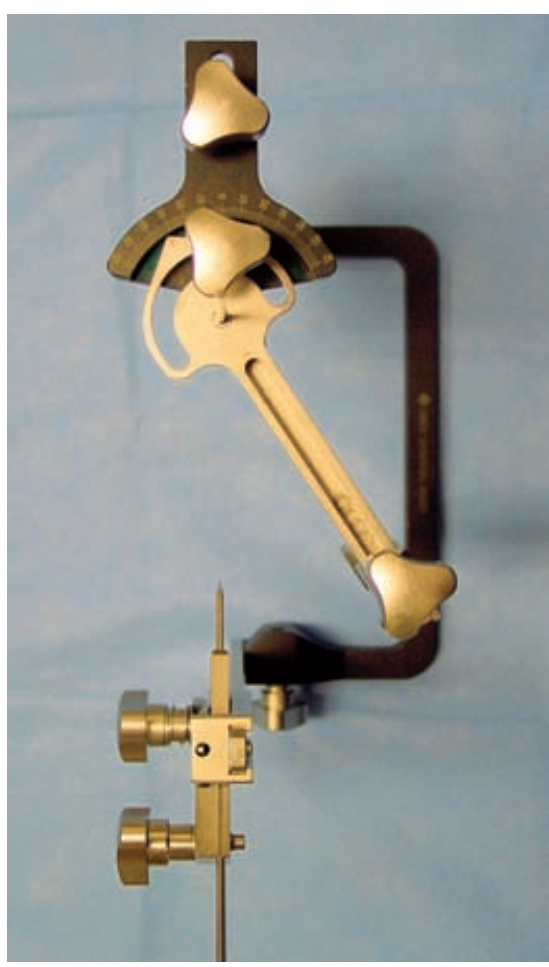

Abb.9 Zur Anfertigungen der Osteotomieschnitte ist eine spezielle Sägelehre (Balansys, Fa. Mathys) erhältlich. 

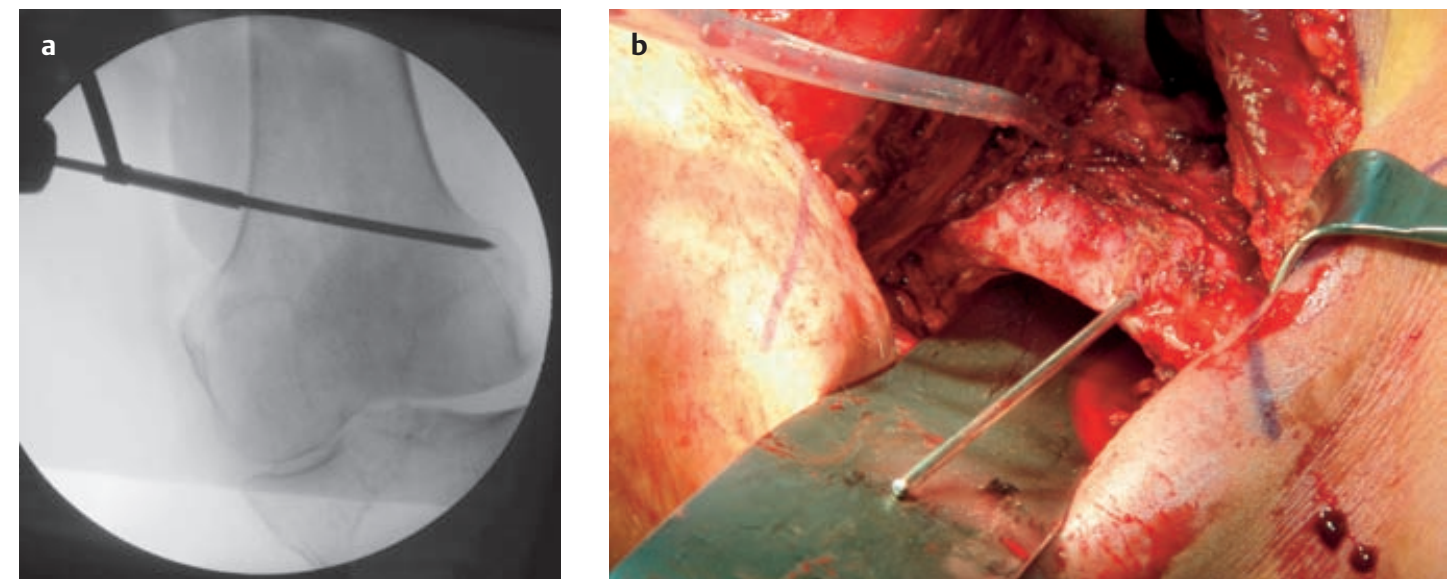

Abb. 10a und b

Alternativ zur Säge-

lehre können die

Osteotomieschnitte auch mit K-Drähten, welche als Führung der ozillierenden Säge dienen, durchgeführt werden.
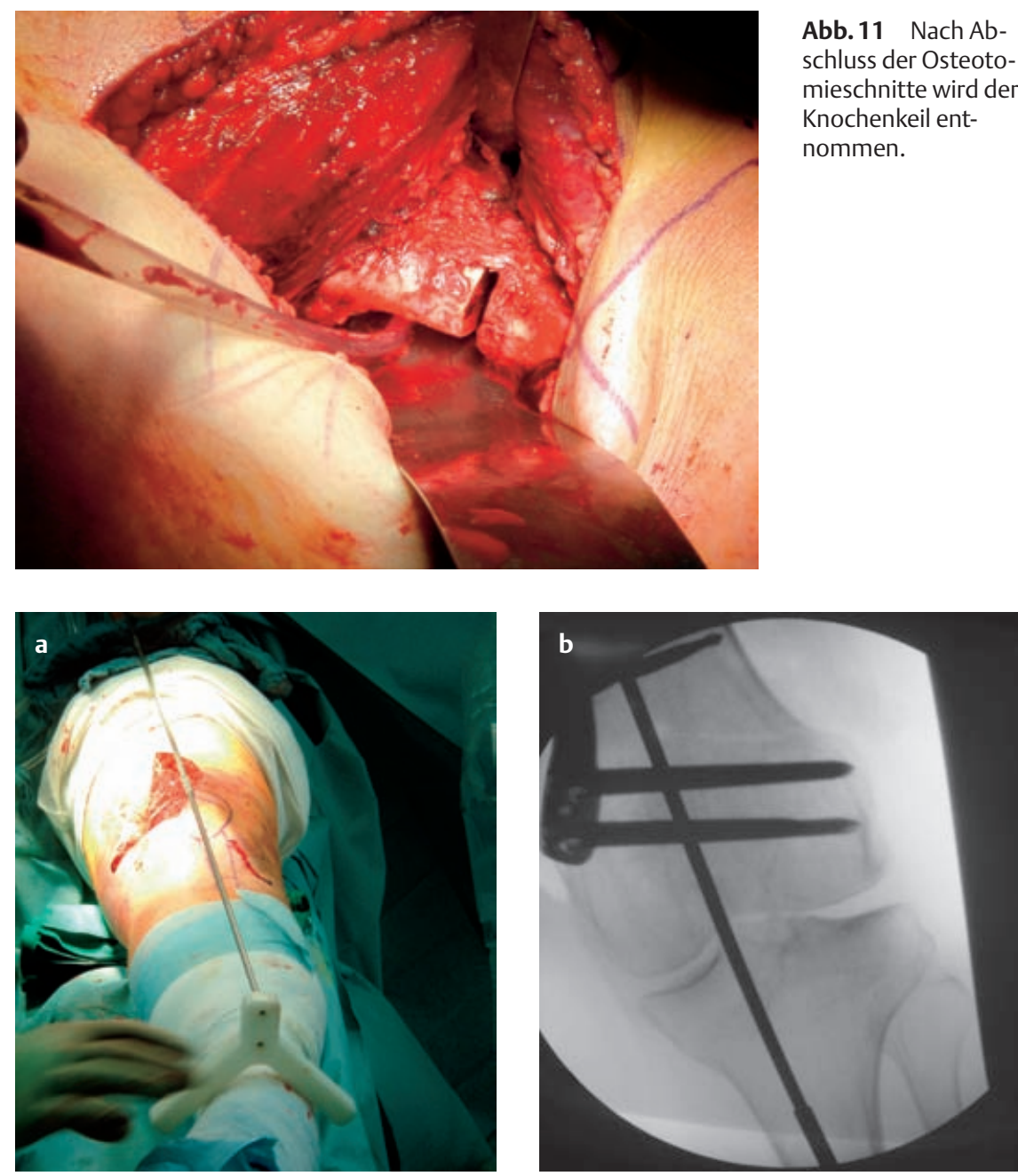

Abb. 12 a und b a Nachdem die Osteotomie geschlossen wurde, wird die mechanische Beinachse mithilfe einer Metallstange überprüft. Diese wir zunächst sowohl über das mittels Bildverstärker ermittelte Hüftkopfzentrum als auch auf die Mitte des oberen Sprunggelenkes gehalten. b Danach kann die Tragachse über dem Kniegelenk abgelesen werden.
Abb.11 Nach Abschluss der Osteotomieschnitte wird der Knochenkeil entnommen.

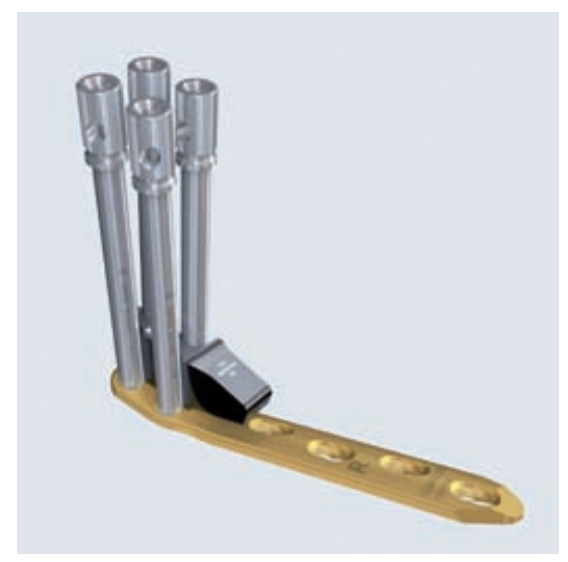

Abb.13 Der TomoFix MDF wird mithilfe einer Positionierungshilfe mit Bohrhülsen besetzt.

von distal unter dem $M$. vastus medialis eingeschoben. Der untere Abschnitt der Platte wird antero-medial auf dem distalen Femur positioniert.

Dabei ist darauf zu achten, dass eine Rechts- und Linksversion der Platte existiert.

Nachdem die Platte korrekt ausgerichtet ist, wird die Position unter Durchleuchtung überprüft. Danach beginnt die Osteosynthese im distalen Abschnitt der Osteotomie. Hierzu werden 5,0 LCP-Kopfverriegelungsschrauben benutzt, welche monokortikal eingebracht werden.

Um Kompression auf die Osteotomie ausüben zu können, kann eine temporäre Zugschraube eingebracht werden (Abb.14a und b). Diese wird im Kombiloch direkt proximal der Osteotomie nicht rechtwinklig zum Schaft, sondern aufsteigend nach proximal lateral eingebracht. Schrittweises Anziehen dieser 

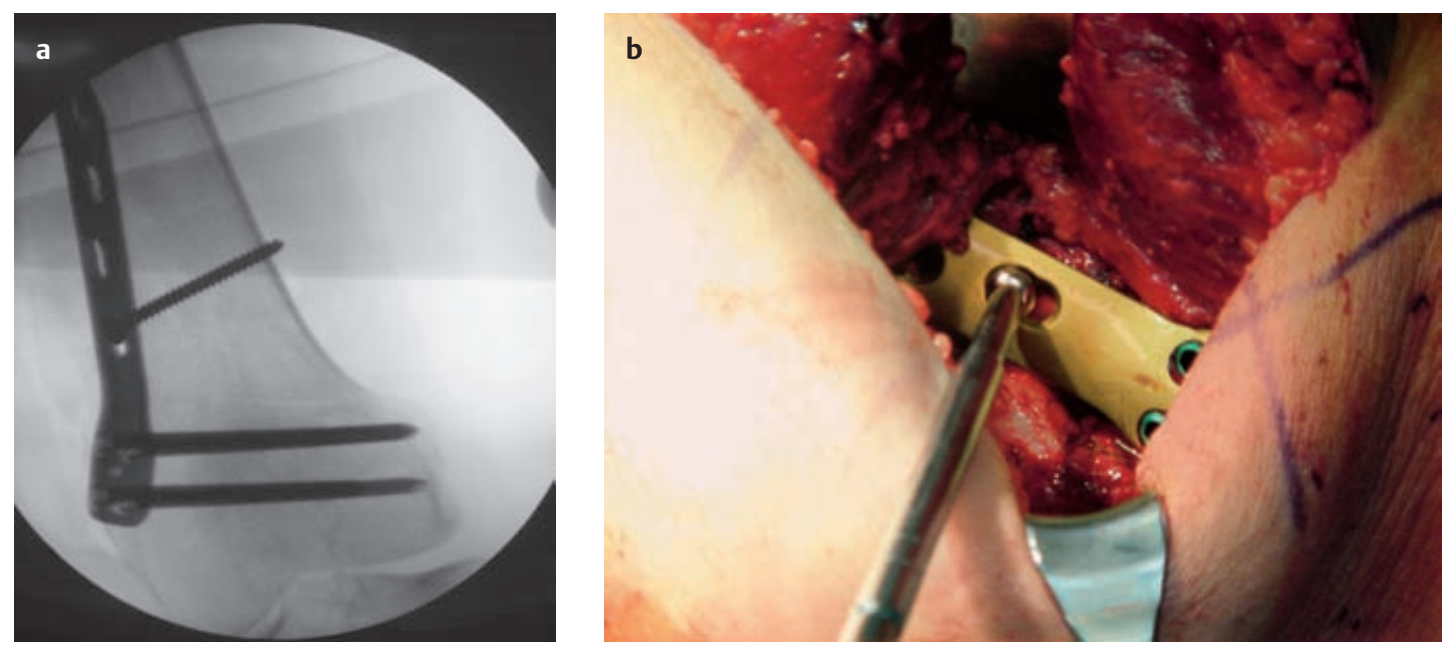

Abb. 14a und b Um Kompression auf die Osteotomie und insbesondere auf die laterale Kortikalis auszuüben, kann eine temporäre Zugschraube eingebracht werden. Die wird im Kombiloch direkt proximal der Osteotomie aufsteigend eingebracht. b Zugschraube in situ.

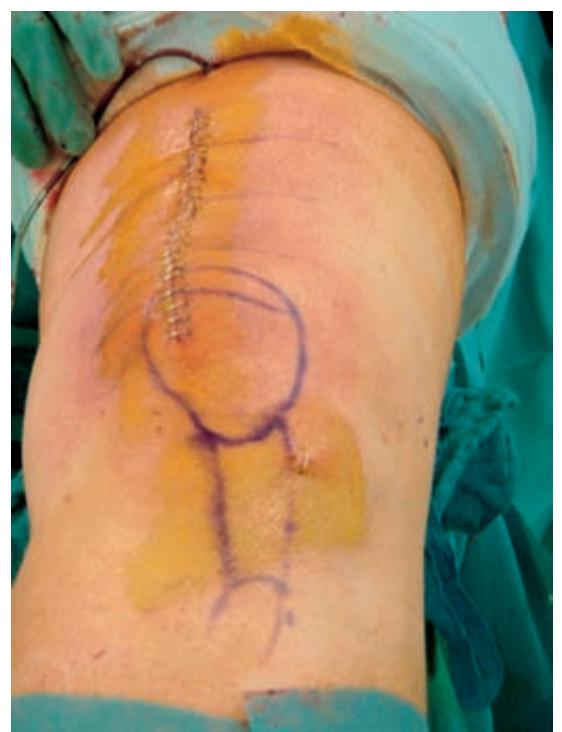

Abb.15 Nach Abschluss der Osteosynthese erfolgt der schichtweise Wundverschluss.

Schraube resultiert in einem nach kaudal gerichteten Kompressionsvektor auf der lateralen Seite des Femurs. Die Schraube sollte sehr vorsichtig unter Durchleuchtungskontrolle angezogen werden. Im Folgenden werden alle Bolzen proximal der Osteotomie eingebracht und die Zugschraube durch einen Bolzen ersetzt. Danach ist die Osteosynthese beendet. Es folgt die Durchleuchtungskontrolle in 2 Ebenen sowie der sorgfältige schichtweise Wundverschluss (Abb.15).

\section{Postoperative Nachbehandlung}

Am Operationstag können bereits vorsichtige Bewegungsübungen bei liegendem Kompressionsverband erfolgen. Um starke Hämatome und Schwellungen zu vermeiden, sollten die Patienten erst am ersten postoperativen Tag aus dem Bett mobilisiert werden. Postoperativ auftre-
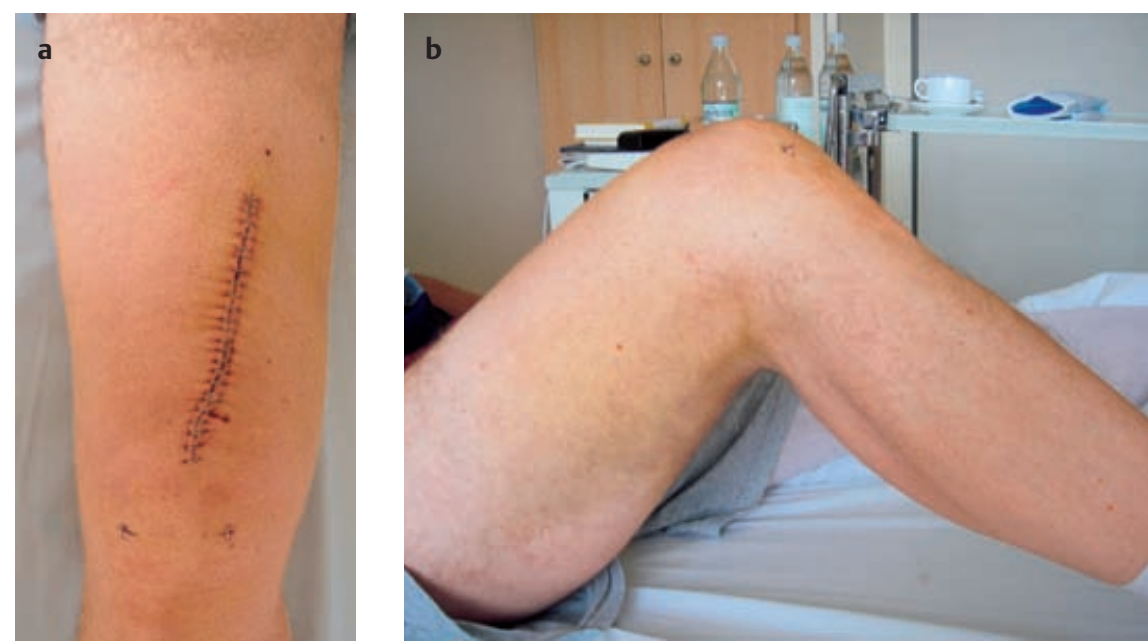

Abb. 16a und b Männlicher, 55-jähriger Patient 4 Tage postoperativ. Das Tragen einer Orthese ist nur in Ausnahmefällen indiziert.

tende Schmerzen können mit Analgetika nach dem WHO-Schema therapiert werden. Der erste Verbandswechsel erfolgt ebenfalls am 1. postoperativen Tag. Im Rahmen dessen wird das angelegte Redon entfernt. Die frühen physiotherapeutischen Behandlungseinheiten umfassen neben den isometrischen Bewegungsübungen ebenfalls die Gangschule an Unterarmgehstützen, wobei eine Belastung von $15-20 \mathrm{~kg}$ nicht überschritten werden sollte. Eine Limitierung des Bewegungsausmaßes ist genauso wie eine stabilisierende Orthese nur in Ausnahmefällen indiziert (Abb.16a und b). Um die postoperativen Lymphödeme und Hämatome zu reduzieren, verwenden wir neben der manuellen Lymphdrainage ebenfalls eine Venenkompressionpumpe. Zur Muskelstimulation hat sich die Verwendung eines EMS-Gerätes (elektrische Muskelstimulation) bewährt.
Diese kann von den Patienten eigenständig durchgeführt werden. Vor der Entlassung, welche bei trockenen und reizlosen Wundverhältnissen etwa 5-7 Tage postoperativ erfolgt, sollte unbedingt eine radiologische Kontrolle erfolgen (Abb.17 a und b).

Die Patienten erhalten zusammen mit den Entlassungspapieren einen detaillierten Nachbehandlungsplan.

Die Teilbelastung sollte für mindestens 6 Wochen eingehalten werden und erst nach erneuter radiologischer Kontrolle bei annähernd knöchern durchbautem Osteotomiespalt schmerzadaptiert gesteigert werden.

Bei noch deutlich einsehbarem Osteotomiespalt sollte die Teilbelastung beibehalten werden. 

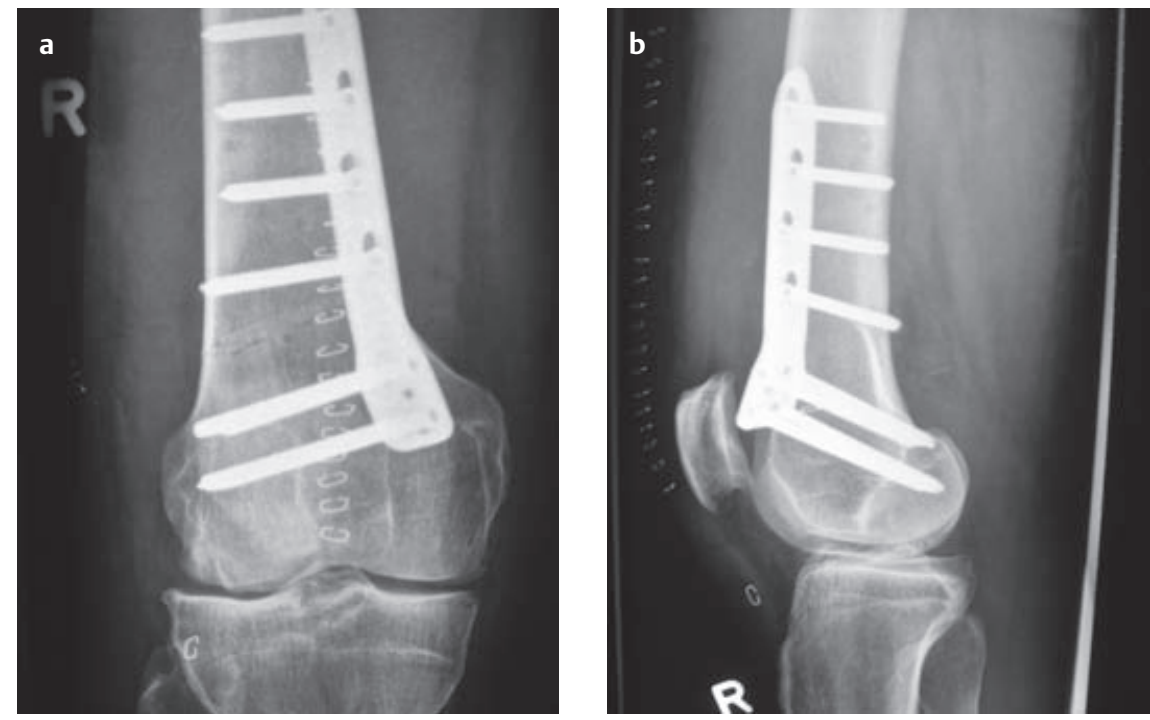

Abb. 17a und $\mathbf{b}$ Vor der Entlassung aus der stationären Behandlung sollte eine radiologische Kontrolle des Operationsergebnisses in 2 Ebenen erfolgen.

\section{Schlussfolgerung}

Die Behandlung von unikompartimentalen Arthrosen des Kniegelenkes in Kombination mit einer valgischen Beinachse war in der Vergangenheit häufig mit hohen Komplikationsraten versehen. In der Literatur werden sowohl verschiedene Operationstechniken als auch unterschiedliche Osteosynthesetechniken beschrieben. In der vorliegenden Arbeit wird eine verbesserte Technik mit einem speziell für diesen Eingriff konzipierten internen Plattenfixateur (TomoFix MDF) beschrieben. Die bisherigen klinischen Ergebnisse sind vielversprechend und geben Anlass zu der Hoffnung, eine sichere und wenig invasive Methode zur operativen Korrektur der Valgusgonarthrose gefunden zu haben.

\section{Literatur}

1 Franco V, Cipolla M, Gerullo G et al. Öffnende Keilosteotomie des distalen Femurs beim Valgusknie. Orthopäde 2004; 33:185-192

2 Galla M, Lobenhoffer P.Die öffnende valgisierende Umstellungsosteotomie der proximalen Tibia mit dem TomoFix ${ }^{\mathrm{TM}}$-Plattenfixateur. Operat Orthop Traumatol 2004; 16:397-417

3 Healy WL, Anglen JO, Wasilewski SA, Krackow KA. Distal femoral varus osteotomy. J Bone Joint Surg 1988; 70:102-109

4 Hankemeier S, Paley D, Pape HC, Zeichen J, Gosling T, Krettek C. Die kniegelenksnahe Focal-dome-Osteotomie. Orthopäde 2004; 33:170-177

5 Lobenhoffer P, Agneskirchner J, Zoch W. Die öffnende valgisierende Osteotomie der proximalen Tibia mit Fixation durch einen medialen Plattenfixateur. Orthopäde 2004; 33:153-160

6 Lobenhoffer P, De Simoni C, Staubli AE. Open wedge high tibial osteotomy with rigid plate fixation. Tech Knee Surg 2002; 1[2]:93-105

7 Outerbridge RE. The etiology of chondromalcia patellae. J Bone Joint Surg 1961; 43B:752757

8 Paley D: Principles of deformity correction. Springer Berlin 2000

9 Pape D, Seil R, Adam et al. Bildgebung und präoperative Planung der Tibiakopfosteotomie. Orthopäde 2004; 3:122-134

10 Stähelin T, Hardegger F. Inkomplette, suprakondyläre Femurosteotomie. Orthopäde 2004; 33:178-184

11 van Heerwaarden R, Wymenga A. Kapitel 9: Die supracondyläre varisierende Femurosteotomie mit speziellem Plattenfixateur In: Lobenhoffer P, Agneskirchner JD, Galla M. Knienahe Umstellungsosteotomien. Thieme Stuttgart 2006 in press

\section{Dr. med. Denise Freiling \\ Assistenzärztin \\ Prof. Dr. med. \\ Philipp Lobenhoffer \\ Chefarzt}

Klinik für Unfall-

und Wiederherstellungschirurgie,

Henriettenstiftung Hannover

Marienstrasse 72-80

30171 Hannover

\section{MD, PhD Ronald van Heerwaarden} Leiter der Abteilung

Klinik für Limb and Deformaty

Reconstruction Unit

Department of Orthopaedics

Martensklinich

Sînt Maartensklinick

Hengstdal 3

6522 JV Nijmegen

Holland 
